\title{
Towards a Dynamical Manin-Mumford Conjecture
}

\section{Dragos Ghioca ${ }^{1}$, Thomas J. Tucker ${ }^{2}$, and Shouwu Zhang ${ }^{3}$}

${ }^{1}$ Department of Mathematics, 1984 Mathematics Road, University of

British Columbia, Vancouver, BC, Canada V6T 1Z2, ${ }^{2}$ Department of Mathematics, Hylan Building, University of Rochester, Rochester, NY 14627, USA, and ${ }^{3}$ Department of Mathematics, Columbia University, 2990 Broadway, New York, NY 10027, USA

Correspondence to be sent to: e-mail: szhang@math.columbia.edu

We provide a family of counterexamples to a first formulation of the dynamical ManinMumford conjecture. We propose a revision of this conjecture and prove it for arbitrary subvarieties of Abelian varieties under the action of group endomorphisms and for lines under the action of diagonal endomorphisms of $\mathbb{P}^{1} \times \mathbb{P}^{1}$.

\section{Introduction}

The Manin-Mumford conjecture, proved by Raynaud [15, 16], states that if $V$ is a subvariety of an Abelian variety $A$, defined over $\mathbb{C}$, then $V$ contains a dense set of torsion points if and only if $V$ is a torsion translate of an Abelian subvariety of $A$. Over number fields, a stronger theorem, conjectured by Bogomolov, and proved by Ullmo [20] and Zhang [25], states that $V$ contains a dense set of points with Néron-Tate height tending to zero if and only if $V$ is a torsion translate of an Abelian subvariety of $A$.

The proofs of Ullmo [20] and Zhang [25] make important use of an equidistribution theorem for points of small canonical height on Abelian varieties (see [19]). Recently, dynamical analogs of this equidistribution theorem have been proved by various authors 
$[2,4,7,21]$. Thus, it seems quite natural to attempt to find dynamical versions of the Bogomolov and Manin-Mumford conjectures.

Here, we will present a family of counterexamples to a first formulation of the dynamical Manin-Mumford and Bogomolov conjectures (see [24, Conjecture 2.5] and [26, Conjecture 1.2.1, Conjecture 4.1.7]). The family is fairly simple. It involves the diagonal subgroup of $E \times E$ under the action of $\left(\left[\omega_{1}\right],\left[\omega_{2}\right]\right)$ for $\left[\omega_{1}\right],\left[\omega_{2}\right] \in \operatorname{End}(E)$ elements of equal degree, where $E$ is an elliptic curve with complex multiplication. This is explained in Theorem 2.2. We then propose a revision of this conjecture in which we show that it holds in the cases of group endomorphisms of Abelian varieties and of lines under the action of arbitrary endomorphisms of $\left(\mathbb{P}^{1}\right)^{N}$.

\section{Counterexamples and Revision}

Our examples are on algebraic varieties, whereas Conjecture 1.2.1 of [26] is a conjecture about more general Kähler varieties; on the other hand, Conjecture 4.1.7 of [26] is a statement about general points of small canonical height, while our counterexample is for the more specific case of preperiodic points (which all have canonical height equal to 0). Thus, for simplicity, we will state an "algebraic dynamical Manin-Mumford" conjecture, which is implied by both (but is weaker than either of) [26, Conjecture 1.2.1] and [26, Conjecture 4.1.7]. We will then provide a family of counterexamples in Theorem 2.2. Before stating the conjecture, we define a few terms:

- An endomorphism $\varphi: X \longrightarrow X$ of a projective variety is said to have a polarization if there exists an ample line bundle $\mathcal{L}$ on $X$ such that $\varphi^{*} \mathcal{L}=\mathcal{L}^{\otimes d}$ for some $d>1$.

- A subvariety $Y$ of a projective variety $X$ with endomorphism $\varphi$ is said to be preperiodic (under the action of $\varphi$ ) if there exist positive integers $m$ and $k$ such that $\varphi^{m+k}(Y)=\varphi^{m}(Y)$.

- $\operatorname{Prep}_{\varphi}=\operatorname{Prep}_{\varphi}(X)$ is defined to be the set of all preperiodic points of $X$ (under the action of $\varphi$ ).

Conjecture 2.1 (Algebraic dynamical Manin-Mumford). Let $\varphi: X \longrightarrow X$ be an endomorphism of a projective variety defined over the complex numbers with a polarization, and let $Y$ be a subvariety of $X$. Then $Y$ is preperiodic if and only if $Y \cap \operatorname{Prep}_{\varphi}(X)$ is Zariski dense in $Y$.

The following theorem shows that Conjecture 2.1 is often false for $E \times E$ when $E$ has complex multiplication. 
Theorem 2.2. Let $E$ be an elliptic curve with complex multiplication defined over the complex numbers. Let $R$ be an order in an imaginary quadratic extension of $\mathbb{Q}$ such that there is an isomorphism $\iota: R \longrightarrow \operatorname{End}(E)$ written as $\iota(\omega)=[\omega]$. Suppose that $\omega_{1}, \omega_{2} \in R$ such that $\left|\omega_{1}\right|=\left|\omega_{2}\right|>1$. Then the diagonal of $E \times E$ is preperiodic under the action of ([$\left.\left.\omega_{1}\right],\left[\omega_{2}\right]\right)$ if and only if $\omega_{1} / \omega_{2}$ is a root of unity.

Remark 2.3. In Theorem 2.2, note that all torsion points of $E$ are preperiodic under both $\left[\omega_{1}\right]$ and $\left[\omega_{2}\right]$ (see also Claim 3.2); therefore, the diagonal subvariety of $E \times E$ has a Zariski dense set of preperiodic points under the map $\left(\left[\omega_{1}\right],\left[\omega_{2}\right]\right)$. Hence Theorem 2.2 gives rise to many counterexamples. For example, take any $\omega_{1}$ such that $\left|\omega_{1}\right|>1$ is the square-root of an odd integer and $\omega_{1} / \bar{\omega}_{1}$ is not a root of unity, and let $\omega_{2}=\bar{\omega}_{1}$. There are also examples in which $\omega_{1}$ is an integer; take for example $\omega_{1}=5$ and $\omega_{2}=3+4 i$ in $\mathbb{Z}[i]$. Note that for each $\omega \in R$, we have $|\omega|^{2}=\# \operatorname{ker}([\omega])=\operatorname{deg}([\omega])$. The lowest degree counterexamples come from multiplication by $\frac{1+\sqrt{-7}}{2}$ on the curve $y^{2}=x^{3}-35 x+98$ (see [18, II.2], where this is worked out in detail). Pazuki [14] has worked out a number of higher-dimensional counterexamples.

We propose the following reformulation of Conjecture 2.1.

Conjecture 2.4. Let $X$ be a projective variety, let $\varphi: X \longrightarrow X$ be an endomorphism defined over $\mathbb{C}$ with a polarization, and let $Y$ be a subvariety of $X$ which has no component included in the singular part of $X$. Then $Y$ is preperiodic under $\varphi$ if and only if there exists a Zariski dense subset of smooth points $X \in Y \cap \operatorname{Prep}_{\varphi}(X)$ such that the tangent subspace of $Y$ at $x$ is preperiodic under the induced action of $\varphi$ on the Grassmanian $\mathrm{Gr}_{\operatorname{dim}(Y)}\left(T_{X, X}\right)$. (Here, we denote by $T_{X, X}$ the tangent space of $X$ at the point $X$.)

One direction is clear: if $Y$ is preperiodic then it will lift to a preperiodic subvariety of the Grassmanian, which will contain a dense set of preperiodic points, by work of Fakhruddin [6]. The other direction seems more difficult. In the following, we show that Conjecture 2.4 is true in the cases of group endomorphisms of Abelian varieties and of arbitrary endomorphisms of $X=\mathbb{P}^{1} \times \mathbb{P}^{1}$ when $Y$ is a line.

\section{Abelian Varieties}

Theorem 3.1. Conjecture 2.4 holds for group endomorphisms $\varphi$ of Abelian varieties $X$. 
Proof. Clearly, it suffices to prove our result assuming $Y$ is irreducible. We start with a standard result regarding preperiodic points of polarizable algebraic group endomorphisms; we include its proof for completeness.

Claim 3.2. For a polarizable algebraic group endomorphism $\varphi$ of an Abelian variety $X$, we have $\operatorname{Prep}_{\varphi}(X)=X_{\text {tor. }}$.

Proof of Claim 3.2. Clearly, each torsion point of $X$ is preperiodic under $\varphi$ since $\varphi$ is an algebraic group endomorphism, and so, for each $m \in \mathbb{N}$, we have $\varphi(X[m]) \subset X[m]$. Since $X[m]$ is a finite set, we conclude that each torsion point in $X[m]$ is preperiodic under $\varphi$.

Conversely, if $\alpha \in \operatorname{Prep}_{\varphi}(X)$, then there are $k>0$ and $m \geq 0$ such that $\varphi^{m}(\alpha)=$ $\varphi^{m+k}(\alpha)$ or $\left(\varphi^{k+m}-\varphi^{m}\right)(\alpha)=0$, or $\alpha$ is in the kernel of $\varphi^{k+m}-\varphi^{m}$. Since $\varphi$ is polarized, then $\varphi$ acts on $\operatorname{Lie}(X)$ with eigenvalues the absolute values of which are $|\operatorname{deg} \varphi|^{1 / 2 \operatorname{dim} X}$. Thus $\left(\varphi^{k+m}-\varphi^{m}\right)$ has all eigenvalues nonvanishing on $\operatorname{Lie}(X)$. It follows that $\varphi^{k+m}-\varphi^{m}$ is finite over $X$ and then has finite kernel. Thus $\alpha$ is torsion.

Now, assume $Y \subset X$ contains a Zariski dense set of points from $\operatorname{Prep}_{\varphi}(X)$. By Claim 3.2, it follows that $Y$ contains a Zariski dense set of torsion points of $X$. Hence, the classical Manin-Mumford conjecture (proved by Raynaud [16]) implies that $Y$ is a torsion translate of an algebraic subgroup of $X$, that is, $Y=\gamma+H$, where $\gamma \in X_{\text {tor }}$ and $H$ is an Abelian subvariety of $X$. Since $\gamma \in \operatorname{Prep}_{\varphi}(X)$ (also according to Claim 3.2), we are left to show that $H$ is preperiodic under $\varphi$.

Now, using the exponential uniformization map on $X$, we see that the preperiodicity of $H$ under the endomorphism $\varphi$ is equivalent with the preperiodicity of the tangent subspace of $H$ at any point $x \in H$ under the induced action of $\varphi$ on $\operatorname{Gr}_{\operatorname{dim}(Y)}\left(T_{X, X}\right)$. This concludes our proof.

Proof of Theorem 2.2. The action of $\left[\omega_{1}, \omega_{2}\right]$ on

$$
\operatorname{Lie}(E \times E)=\operatorname{Lie}(E) \oplus \operatorname{Lie}(E)
$$

is given as a multiplication by $\left(\omega_{1}, \omega_{2}\right)$. The diagonal in $\operatorname{Lie}(E) \oplus \operatorname{Lie}(E)$ is preperiodic under $\left(\omega_{1}, \omega_{2}\right)$ if and only if $\omega_{1} / \omega_{2}$ is a root of unity.

\section{Lines in $\mathbb{P}^{1} \times \mathbb{P}^{1}$}

Theorem 4.1. Let $f, g: \mathbb{P}^{1} \longrightarrow \mathbb{P}^{1}$ be nonconstant rational maps over $\mathbb{C}$ such that $\operatorname{deg} f=$ $\operatorname{deg} g>1$, let

$$
(f, g): \mathbb{P}^{1} \times \mathbb{P}^{1} \longrightarrow \mathbb{P}^{1} \times \mathbb{P}^{1}
$$


be the map given by $(f, g)(a, b)=(f(a), g(b))$, and let $L \subset \mathbb{P}^{1} \times \mathbb{P}^{1}$ be a line. If there exists an infinite subset of points $x \in \operatorname{Prep}_{(f, g)} \cap L$ such that the tangent subspace of $L$ at $x$ is preperiodic under the induced action of $\varphi$ on $\mathrm{Gr}_{1}\left(T_{\mathbb{P}^{1} \times \mathbb{P}^{1}, X}\right)$, then $L$ is preperiodic under $(f, g)$.

The proof is somewhat similar to the proof of the main result from [8] which dealt with the case of polynomial maps.

Step 1: reduction to the case $L$ is the diagonal subvariety. If $L$ does not project dominantly to both copies of $\mathbb{P}^{1}$, then (without loss of generality) we may assume $L=\{a\} \times \mathbb{P}^{1}$. Since $L$ contains infinitely many preperiodic points for the action of $(f, g)$ on $\mathbb{P}^{1} \times \mathbb{P}^{1}$, we conclude that $a$ is preperiodic for $f$. Therefore, $L$ is indeed preperiodic under the action of $(f, g)$ (no extra hypothesis is necessary in this case).

So, from now on, we assume $L$ projects dominantly on $\mathbb{P}^{1} \times \mathbb{P}^{1}$. Thus, there exists a linear map $\sigma$ such that the equation of $L$ is $y=\sigma(x)$, where $(x, y)$ is a generic pair of projective coordinates for $\mathbb{P}^{1} \times \mathbb{P}^{1}$. Then, replacing $g$ by $\sigma^{-1} \circ g \circ \sigma$ yields that the diagonal line $\Delta$ contains infinitely many preperiodic points for $\left(f, \sigma^{-1} g \sigma\right)$. Therefore, from now on, we assume $L=\Delta$ is the diagonal subvariety of $\mathbb{P}^{1} \times \mathbb{P}^{1}$.

Step 2: using theorems of equidistribution in dynamics. First we note that $(x, x) \in \Delta$ is in $\operatorname{Prep}_{(f, g)}$ if and only if $x \in \operatorname{Prep}_{f} \cap \operatorname{Prep}_{g}$. Thus, $\operatorname{Prep}_{(f, g)} \cap \Delta$ is infinite if and only if $\operatorname{Prep}_{f} \cap$ Prep $_{g}$ is infinite. We denote the equilibrium measure and the Julia set of a map $h$ of degree greater than 1 as $\mu_{h}$ and $\mathcal{J}_{h}$, respectively. By recent work of Baker and DeMarco [1] and Yuan and Zhang [22], we have Prep ${ }_{f}=\operatorname{Prep}_{g}, \mu_{f}=\mu_{g}$ and $\mathcal{J}_{f}=\mathcal{J}_{g}$.

Step 3: applying a theorem of Levin. Thus, $f$ and $g$ satisfy the conditions (i) and (ii) of Theorem 4.2 proved in [12] (see also [5]).

Theorem 4.2 (Levin). Let $f, g \in \mathbb{C}(t)$ be rational maps of degree greater than 1 satisfying the following two conditions:

(i) $\mathcal{J}_{f}=\mathcal{J}_{g}$ and $\mu_{f}=\mu_{g}$.

(ii) There exists a repelling periodic point for $g$ which is also preperiodic for $f$.

Then one of the following conclusions hold:

(1) There exist $k, \ell \in \mathbb{N}$ such that $f^{\ell} \circ g^{k}=f^{2 \ell}$. 
(2) Modulo conjugation, $f$ and $g$ are either
(a) $t^{m}$ for some $m \in \mathbb{Z}$; or
(b) $T_{m}$ for some $m \in \mathbb{N}$ (where $T_{m}$ denotes the $m$ th Chebychev polynomial); or
(c) a Lattès map.

Assume first that (1) holds. Using the fact that $\operatorname{deg}(f)=\operatorname{deg}(g) \geq 2$, we conclude that $k=\ell$ in conclusion (1) of Theorem 4.2. Hence $f^{\ell} \circ g^{\ell}=f^{2 \ell}$. We claim that this relation yields that the diagonal $\Delta \in \mathbb{P}^{1} \times \mathbb{P}^{1}$ is $(f, g)$-preperiodic.

Indeed, because $g^{\ell}: \mathbb{P}^{1} \longrightarrow \mathbb{P}^{1}$ is surjective, for each $x \in \mathbb{P}^{1}(\mathbb{C})$, there exists $y \in \mathbb{P}^{1}(\mathbb{C})$ such that $x=g^{\ell}(y)$. Furthermore, using that $f^{\ell} \circ g^{\ell}=f^{2 \ell}$, we obtain that $f^{2 \ell}(y)=f^{\ell}\left(g^{\ell}(y)\right)$, and so, $\left(f^{2 \ell}(y), g^{2 \ell}(y)\right)=\left(f^{\ell}(x), g^{\ell}(x)\right)$. Therefore, $\left(f^{\ell}, g^{\ell}\right)(\Delta) \subset$ $\left(f^{2 \ell}, g^{2 \ell}\right)(\Delta)$. Moreover, since $\Delta$ is irreducible, we conclude that $\left(f^{2 \ell}, g^{2 \ell}\right)(\Delta)=\left(f^{\ell}, g^{\ell}\right)(\Delta)$, and thus $\Delta$ is $(f, g)$-preperiodic, as conjectured. Note that so far we did not need the extra assumption about the preperiodicity of the tangent subspaces.

Step 4: the case when $f$ and g satisfy conclusion (2) in Theorem 4.2. Now, in the case where one of the maps is conjugate to a powering map or a Chebychev polynomial (cases (a) and (b) above), the other cannot be Lattès map since the two maps must have the same Julia set; the Julia set of a Lattès map is the entire Riemann sphere, which can never be the case for a polynomial or for a rational map $t^{m}$ for $m \in \mathbb{Z}$. Similarly, if one map is conjugate to $t^{m}$, then the other one cannot be conjugate to $T_{m}$ (and vice-versa) since the Julia set of $t^{m}$ is the unit circle, while the Julia set for $T_{m}$ is the closed interval $[-2,2]$. Now, if both $f$ and $g$ are conjugate to $t^{m}$ (which is an endomorphism of $\mathbb{G}_{m}$ ), then our problem reduces to the classical Manin-Mumford question for $\mathbb{G}_{m}^{2}$ which was first proved by Lang [11] (see also the results of Raynaud [15, 16] for the general case of Abelian varieties). A similar argument yields the desired result if both $f$ and $g$ are conjugate to $T_{m}$.

Therefore, we reduced the proof of Theorem 4.1 to the case when both $f$ and $g$ are Lattès maps. In this case, there exist two elliptic curves $E_{1}$ and $E_{2}$, there exist two projection maps $\pi_{i}: E_{i} \longrightarrow \mathbb{P}^{1}$ for $i=1,2$, and there exist two isogenies $\omega_{i}: E_{i} \longrightarrow E_{i}$ witnessing the fact that $f$ and $g$ are Lattès maps, that is,

$$
\pi_{1} \circ \omega_{1}=f \circ \pi_{1} \text { and } \pi_{2} \circ \omega_{2}=g \circ \pi_{2} .
$$

For each unramified point $x \in E_{1} \times E_{2}$ for the morphism $\left(\pi_{1}, \pi_{2}\right)$, the induced map on the tangent space is an isomorphism. Now, the ramified locus for $\left(\pi_{1}, \pi_{2}\right)$ is a 
finite union of divisors of $E_{1} \times E_{2}$ of the form $\{a\} \times E_{2}$ or $E_{1} \times\{b\}$. On the other hand, each irreducible component of $\left(\pi_{1}, \pi_{2}\right)^{-1}(\Delta)$ has dominant projection onto each $E_{i}$ for $i=1,2$. Therefore, for each irreducible component $V$ of $\left(\pi_{1}, \pi_{2}\right)^{-1}(\Delta)$, there exist at most finitely many ramified points for the map $\left(\pi_{1}, \pi_{2}\right)$. Also, for each $(x, x) \in \operatorname{Prep}_{(f, g)}\left(\mathbb{P}^{1} \times \mathbb{P}^{1}\right)$ each point in $\left(\pi_{1}, \pi_{2}\right)^{-1}(x, x) \cap V$ is preperiodic for the action of $\Phi:=\left(\omega_{1}, \omega_{2}\right)$ on $E_{1} \times E_{2}$. Since there are only finitely many ramified points of $\left(\pi_{1}, \pi_{2}\right)$ lying on $V$, and because $\left(\pi_{1}, \pi_{2}\right)(V)=\Delta$, we obtain that there are infinitely many preperiodic points $x$ under $\Phi$ lying on $V$ such that the tangent subspace of $V$ is preperiodic under the induced action of $\Phi$ on $\mathrm{Gr}_{1}\left(T_{E_{1} \times E_{2}, X}\right)$. Because Conjecture 2.4 holds for algebraic group endomorphisms of Abelian varieties, we conclude that $V$ is preperiodic under $\Phi$; therefore $\Delta$ is preperiodic under $(f, g)$ as desired.

Remark 4.3. It is worth noting that if not both $f$ and $g$ are Lattès maps, then we actually get that any line $L \subset \mathbb{P}^{1} \times \mathbb{P}^{1}$ is preperiodic once it contains infinitely many preperiodic points; we do not need in this case the assumption about the preperiodicity of the tangent subspaces. We only need this last assumption when both $f$ and $g$ are Lattès maps.

Remark 4.4. We can extend Theorem 4.1 in a number of ways:

(a) Over number fields, Theorem 4.1 also holds under the weaker "Bogomolov type" condition that there is an infinite nonrepeating sequence of points $\left(\alpha_{i}\right) \in \mathbb{P}^{1}(\overline{\mathbb{Q}})$ such that

$$
\lim _{i \rightarrow \infty} h_{f}\left(\alpha_{i}\right)=\lim _{i \rightarrow \infty} h_{g}\left(\alpha_{i}\right)=0
$$

This follows immediately from the fact that the equidistribution results of $[2,4,7,21]$ apply to such families of points.

(b) Levin [12] proved that if $f$ and $g$ do not satisfy conclusion (1) of Theorem 4.2, then actually $f$ and $g$ share a common parabolic orbifold (see [12] for its definition). Using the work of Douady-Hubbard-Thurston [5] one obtains then the precise formulation from condition (2) of Theorem 4.2. This fact is proved in [5] in a general topological setting. It can be proved much more simply for rational functions, using Galois theory (see [9]). For example, consider the parabolic orbifold that arises when $f$ is a degree 4 map which maps six points with ramification index 2 onto three points in its image. Taking a 
genus 1 cover of $\mathbb{P}^{1}$ that ramifies at exactly these three points plus one more point then gives rise to the multiplication-by-2 map on an elliptic curve that descends to $f$ under the hyperelliptic involution (this can be checked easily using Abhyankar's lemma for ramification indices under composita).

(c) Theorem 4.1 extends easily to the case of any line in $\left(\mathbb{P}^{1}\right)^{N}$ under the action of $\left(f_{1}, \ldots, f_{N}\right)$ for arbitrary rational maps $f_{i}$; the proof in this case is reduced to the case $N=2$ by projecting on any set of two coordinates of $\left(\mathbb{P}^{1}\right)^{N}$.

The proof of Theorem 4.1 can be generalized to give a complete answer to Questions 29(1) and (2) of [10] in the case of rational functions defined over the complex numbers.

Question 4.5 (Kawaguchi-Silverman). Let $f, g \in \mathbb{C}(x)$ be rational maps of degree at least equal to 2 .

(1) If Prep $_{f}=$ Prep $_{g}$, then how are $f$ and $g$ related?

(2) If there is an infinite set $Z \subset$ Prep $_{f}$ with the property that $g(Z) \subset$ Prep $_{f}$, then how are $f$ and $g$ related?

We note that the first question above is easier than the second, because Prep $f$ always is infinite, and if $\operatorname{Prep}_{f}=\operatorname{Prep}_{g}$, then $g\left(\operatorname{Prep}_{f}\right)=\operatorname{Prep}_{f}$. We will use Theorem 4.1 to answer Question 4.5 (2). This immediately gives an answer to Question 4.5 (1) as well.

Corollary 4.6. If $f$ and $g$ are two rational functions such that there exists an infinite set $Z \subset \operatorname{Prep}_{f}$ with the property that $g(Z) \subset \operatorname{Prep}_{f}$, then either $f^{k} g^{\ell}=f^{2 k}$ for some positive integers $\ell$ and $k$, or $f$ and $g$ are both Chebychev, powering, or Lattès maps, modulo conjugation by linear maps.

Proof. We will prove that the invariant metrics $\|\cdot\|_{f}$ and $\|\cdot\|_{g}$ on $O(1)$ defined by $f$ and $g$, respectively, are proportional. This will allow us to use Theorem 4.2.

Let $K$ be the subfield of $\mathbb{C}$ generated by the coefficients of $f$ and $g$ and $\bar{K}$ the algebraic closure of $K$ in $\mathbb{C}$, then $f$ and $g$ are the base changes of endomorphisms $f_{K}, g_{K}$ of $\mathbb{P}_{K}^{1}$, and $Z$ is the base change of a subset $Z_{K}$ in $\operatorname{Prep}_{f}(\bar{K})$. With $Z_{K}$ replaced by the union of its conjugates under $\operatorname{Gal}(\bar{K} / \mathrm{K})$, we may assume that $Z_{K}$ is a union of closed points in $\mathbb{P}_{K}^{1}$. Now we further extend $f_{K}, g_{K}$ to endomorphisms

$$
f_{U}, g_{U}: \mathbb{P}_{U}^{1} \longrightarrow \mathbb{P}_{U}^{1}
$$


over a variety $U$ over $\mathbb{Q}$ of finite type and with function field $K$. For each valuation $v$ of $K$, the bundle $O(1)$ has invariant metrics $\|\cdot\|_{v, f_{U}}$ and $\|\cdot\|_{v, g_{U}}$ defined for $f_{U}$ and $g_{U}$ on the Berkovich space $X_{v}^{a n}$. Let $Z_{U}$ be the union of Zariski closure of closed points in $Z_{K}$. For each geometric point $t \in U(\overline{\mathbb{Q}})$, the objects $\left(f_{U}, g_{U}, Z_{U}\right)$ have reductions $\left(f_{t}, g_{t}, Z_{t}\right)$ such that $Z_{t} \in \operatorname{Prep}_{f_{t}}$ with $g_{t}\left(Z_{t}\right) \subset \operatorname{Prep}_{f_{t}}$. By [23, Theorem 4.7], $Z_{t}$ is still infinite.

Applying [13, Theorem 3.5] to the graph of $g$ in $\mathbb{P}^{1} \times \mathbb{P}^{1}$, we see that for all $x \in \operatorname{Prep}_{f_{t}}$ we have $g_{t}(x) \in \operatorname{Prep}_{f_{t}}$. Therefore $g_{t}\left(\operatorname{Prep}_{f_{t}}\right) \subset \operatorname{Prep}_{f_{t}}$. By [22], this implies that the invariant adelic metrics $\|\cdot\|_{f_{t}}$ and $\|\cdot\|_{g_{t}}$ on $O(1)$ defined by $f_{t}$ and $g_{t}$, respectively, are proportional. In particular, this is true for all $t \in U(\overline{\mathbb{Q}})$ and for the Archimedean place corresponding to $\overline{\mathbb{Q}} \subset \bar{K} \subset \mathbb{C}$. By continuity of $\|\cdot\|_{f_{U}}$ and $\|\cdot\|_{g_{U}}$, this is true for all points in $U(\mathbb{C})$ including the point corresponding to original embedding $K \subset \mathbb{C}$. Thus, we have shown that the invariant metrics $\|\cdot\|_{f}$ and $\|\cdot\|_{g}$ are proportional. This implies that Prep $_{f}=\operatorname{Prep}_{g}, \mu_{f}=\mu_{g}$ and $\mathcal{J}_{f}=\mathcal{J}_{g}$; an application of Theorem 4.2 finishes our proof.

\section{Other Questions}

In this section, we explore other questions related to the Dynamical Manin-Mumford problem. Each time we show that our question is valid for both polarizable group endomorphisms of Abelian varieties, and for lines under the action of polarizable endomorphisms of $\left(\mathbb{P}^{1}\right)^{m}$ for any $m \in \mathbb{N}$.

Our first question refers to a classical principle in arithmetic geometry: for a given ambient variety $X$, one defines the notion of being special both for points and for irreducible subvarieties of $X$. Generically, one expects that if an irreducible subvariety $Y$ of $X$ contains a Zariski dense set of special points, then $Y$ is a special subvariety. This principle lies at the heart of the classical Manin-Mumford conjecture-in that case, the ambient variety is an Abelian variety $G$, the special points are the torsion points of $G$, while the special irreducible subvarieties are torsion translates of Abelian subvarieties of G. For the Algebraic Dynamical Manin-Mumford Conjecture (see Conjecture 2.1), the ambient variety is any projective variety endowed with a polarizable endomorphism $\Phi$, the special points are the preperiodic points for $\Phi$, and the special irreducible subvarieties of $X$ are the preperiodic irreducible subvarieties of $X$. Our counterexample from Section 2 shows that an irreducible subvariety may contain a Zariski dense set of special points (in the algebraic dynamics context) without being itself a special subvariety. However, it is natural to raise the following question regarding subvarieties containing a Zariski dense set of preperiodic points. 
Question 5.1. Let $X$ be a projective variety and let $\Phi: X \longrightarrow X$ be a polarizable endomorphism defined over the complex numbers. Assume $X_{1}$ and $X_{2}$ are any two subvarieties of $X$ containing a Zariski dense set of preperiodic points under $\Phi$. Must $X_{1} \cap X_{2}$ contain a Zariski dense set of preperiodic points under $\Phi$ ?

In particular, our Question 5.1 asks whether two (distinct) irreducible curves containing infinitely many preperiodic points must intersect in finitely many preperiodic points.

Question 5.1 has a positive answer when $X$ is an Abelian variety and $\Phi$ is a polarizable algebraic group endomorphism. Indeed, in that case the preperiodic points for $\Phi$ are the torsion points of $X$ (see Claim 3.2), and we know (by the classical ManinMumford conjecture proved by Raynaud $[15,16])$ that any irreducible subvariety of $X$ which contains a Zariski dense set of torsion points must be a torsion translate of an Abelian subvariety of $X$ (note also that the torsion points are dense in each Abelian subvariety).

We also have a positive answer for Question 5.1 when $X=\left(\mathbb{P}^{1}\right)^{m}$ (for some $m \in \mathbb{N}$ ) and $X_{1}$ and $X_{2}$ are lines. Indeed, first we may reduce to the case $m=2$ as observed in Remark 4.4 (c). Secondly, our proof of Theorem 4.1 shows that if $Y \subset \mathbb{P}^{1} \times \mathbb{P}^{1}$ is a line which contains infinitely many preperiodic points for $\Phi$, then either $Y$ is preperiodic under $\Phi$, or $\Phi=(f, g)$ for two Lattès maps $f$ and $g$ (see Remark 4.3). So, if $\Phi$ is not given by the action of two Lattès maps, we conclude that both lines $X_{1}$ and $X_{2}$ satisfying the hypothesis of Question 5.1 are preperiodic under $\Phi$, and thus their point of intersection is also preperiodic under $\Phi$. Now, if $\Phi=(f, g)$ and $f$ and $g$ are Lattès maps, then exactly as in the proof of Theorem 4.1 we may reduce the question from $\mathbb{P}^{1} \times \mathbb{P}^{1}$ to a question on a split Abelian surface. The reasoning from the above paragraph regarding Abelian varieties finishes the proof for Question 5.1 for lines in $\mathbb{P}^{1} \times \mathbb{P}^{1}$.

A positive answer to Question 5.1 would have the following interesting consequence. Using the automatic uniformity theorem proved by Scanlon [17], we deduce that the set of preperiodic points of $\Phi$ satisfies automatic uniformity (since the hypothesis of Theorem 2.4 from [17] is implied by a positive answer to Question 5.1). Therefore, for each positive integer $d$, there exists an integer $B(d)$ (depending on $d, X$ and $\Phi$ only) such that for any subvariety $Y$ contained in $X$ of degree at most $d$ (computed with respect to a fixed embedding of $X$ into the projective space as given by the polarization), if $Y \cap \operatorname{Prep}_{\Phi}(X)$ is not Zariski dense in $Y$, the Zariski closure of $Y \cap \operatorname{Prep}_{\Phi}(X)$ has at most $B(d)$ irreducible components. In particular, if $Y \cap \operatorname{Prep}_{\Phi}(X)$ is a finite set, then $\left|Y \cap \operatorname{Prep}_{\Phi}(X)\right| \leq B(d)$. 
Remark 5.2. It is worth noting that the hypothesis in [17, Theorem 2.4] is that if $X_{1}$ and $X_{2}$ contain a Zariski dense set of special points, then each irreducible component of $X_{1} \cap X_{2}$ which contains at least one special point must contain a Zariski dense set of special points. Hence, one may consider a weakening of our Question 5.1 by asking that if $X_{1}$ and $X_{2}$ contain a Zariski dense set of preperiodic points for the endomorphism $\Phi$, then each irreducible component of $X_{1} \cap X_{2}$ which contains at least one preperiodic point for $\Phi$ must contain a Zariski dense set of preperiodic points for $\Phi$.

Remark 5.3. Phrased in terms of canonical heights of varieties, Question 5.1 asks whether $X_{1} \cap X_{2}$ must have canonical height zero if $X_{1}$ and $X_{2}$ each have canonical height zero, via results of [24]. By the Bost-Gillet-Soulé arithmetical Bézout theorem [3], one has the bound

$$
h\left(X_{1} \cdot X_{2}\right) \leq\left(\operatorname{deg} X_{1}\right) h\left(X_{2}\right)+\left(\operatorname{deg} X_{2}\right) h\left(X_{1}\right)+c\left(\operatorname{deg} X_{1}\right)\left(\operatorname{deg} X_{2}\right)
$$

for some nonnegative number $c$, where $h$ is the height on $\mathbb{P}^{n}$ coming from the FubiniStudy metric and $X_{1}, X_{2}$ are two subvarieties that intersect properly. One might hope to show that the extra "error term" $c\left(\operatorname{deg} X_{1}\right)\left(\operatorname{deg} X_{2}\right)$ is zero when the height $h$ is the canonical height for a polarizable dynamical system, which would thus provide a positive answer to our Question 5.1. Indeed, Bost-Gillet-Soulé state that they believe this "error term" is zero for the Fubini-Study metric and that this can be proved for linear subvarieties (see [3, p. 905]). However, Pascal Autissier has shown us that there is indeed such an error term for the canonical height associated with a polarized dynamical system even for lines with respect to the canonical height coming from the squaring map on $\mathbb{P}^{2}$, so a different approach to Question 5.1 is needed. More precisely, let $n$ be any integer larger than 1 , let $L_{1}$ be the line given by the equation $n x-(n-1) y=0$, and let $L_{2}$ be the line given by the equation $n x+(n-1) y-z=0$. Since for points in $\mathbb{P}^{2}$, the canonical height associated with the squaring map is the usual (naive) Weil height, and since $L_{1} \cdot L_{2}=[n-1: n: 2 n(n-1)]$, we conclude that $h\left(L_{1} \cdot L_{2}\right)=\log (2 n(n-1))$. Using the Mahler measure one obtains $h\left(L_{1}\right)=h\left(L_{2}\right)=\log (n)$, and so, $h\left(L_{1} \cdot L_{2}\right)=h\left(L_{1}\right)+h\left(L_{2}\right)+$ $\log (2 \cdot(1-1 / n))$. Hence there exists an error term in (5.1) for the canonical height associated with the squaring map. The best error term for the naive height on $\mathbb{P}^{2}$ should be $\log (2)$, that is, the above examples are most likely asymptotically sharp.

We may also ask the following natural question which connects the dynamical Manin-Mumford and the Dynamical Bogomolov Conjectures in the context of algebraic 
dynamics (note that this connection is already known in the case of the classical Bogomolov and Manin-Mumford Conjecture for Abelian varieties).

Question 5.4. Let $X$ be a projective variety over a number field, and let $\Phi$ be a polarizable endomorphism of $X$ defined over $\overline{\mathbb{Q}}$. We denote by $\hat{h}$ the canonical height associated with $\Phi$, and for each $\epsilon>0$ we define

$$
X_{\epsilon}:=\{x \in X(\overline{\mathbb{Q}}): \hat{h}(x)<\epsilon\} .
$$

Then a subvariety $Y$ of $X$ must contain a Zariski dense set of preperiodic points for $\Phi$ if and only if for each $\epsilon>0$ the subset $X_{\epsilon} \cap Y$ is Zariski dense in $Y$ ?

Since the preperiodic points for $\Phi$ are precisely the points of canonical height 0 , we immediately note that once a subvariety $Y$ contains a Zariski dense set of preperiodic points, then $Y \cap X_{\epsilon}$ is Zariski dense in $Y$.

Our proof of Theorem 4.1 yields a positive answer to Question 5.4 for lines embedded in $\left(\mathbb{P}^{1}\right)^{m}$ for any $m \in \mathbb{N}$. Indeed, as explained in Remark 4.4(c), one reduces the question to lines $Y$ inside $\mathbb{P}^{1} \times \mathbb{P}^{1}$ under the action of $\Phi=(f, g)$, and then we have two cases. If not both $f$ and $g$ are Lattès maps, then $Y$ is preperiodic as long as it contains infinitely many points of small height (see Remark 4.4(a)). If both $f$ and $g$ are Lattès maps, then arguing as in the proof of Theorem 4.1 our problem reduces to Question 5.4 for Abelian varieties, which we already know holds.

\section{Acknowledgments}

We would like to thank Pascal Autissier, Philipp Habegger, Fabien Pazuki, Thomas Scanlon, Lucien Szpiro, Xinyi Yuan, and Michael Zieve for many helpful conversations, and we thank the referee for many useful suggestions. We would also like to thank the Centro di Ricerca Matematica Ennio De Giorgi and the University of Bordeaux 1 for their hospitality.

\section{Funding}

This work was partially supported by NSERC (to D.G.) and by NSF Grants 0801072 and 0854839 (to T.J.T.) and a National Science Foundation grant and Guggenheim Fellowship (to S.Z.). 


\section{References}

[1] Baker, M. and L. DeMarco. "Preperiodic points and unlikely intersections." Preprint: arxiv.org/abs/0911.0918.

[2] Baker, M. and R. Rumely. "Equidistribution of small points, rational dynamics, and potential theory." Annales de linstitut Fourier (Grenoble) 56, no. 3 (2006): 625-88.

[3] Bost, J.-B., H. Gillet, and C. Soulé. "Heights of projective varieties and positive green forms." Journal of American Mathematical Society 7, no. 4 (1994): 903-1027.

[4] Chambert-Loir, A. "Mesures et équidistribution sur les espaces de Berkovich." Journal für die Reine und Angewandte Mathematik 595 (2006): 215-35.

[5] Douady, A. and J. H. Hubbard. "A proof of Thurston's topological characterization of rational functions." Acta Mathematica 171, no. 2 (1993): 263-97.

[6] Fakhruddin, N. "Questions on self maps of algebraic varieties." Journal of Ramanujan Mathematical Society 18, no. 2 (2003): 109-22.

[7] Favre, C. and J. Rivera-Letelier. "Équidistribution quantitative des points de petite hauteur sur la droite projective." Mathematische Annalen 335, no. 2 (2006): 311-61.

[8] Ghioca, D. and T. J. Tucker. "Proof of a dynamical Bogomolov conjecture for lines under polynomial actions." Proceedings of the American Mathematical Society 138, no. 2 (2010): 937-42.

[9] Ghioca, D. and M. E. Zieve. "Lattès maps in every characteristic." in preparation.

[10] Kawaguchi, S. and J. H. Silverman. "Dynamics of projective morphisms having identical canonical heights." Proceedings of the London Mathematical Society (3) 95, no. 2 (2007): 519-44.

[11] Lang, S. “Division points on curves." Annali di Matematica Pura ed Applicata (4) 70 (1965): 229-34.

[12] Levin, G. M. “Symmetries on Julia sets." Matematicheskie Zametki 48, no. 5 (1990): 72-79, 159.

[13] Mimar, A. "On the preperiodic points of an endomorphism of $\mathbb{P}^{1} \times \mathbb{P}^{1}$ which lie on a curve." PhD thesis, Columbia University, 1997.

[14] Pazuki, F. "Zhang's conjecture and squares of Abelian surfaces." Comptes Rendus Mathématique Académie des Sciences Paris 348, no. 9-10 (2010): 483-486.

[15] Raynaud, M. “Courbes sur une variété abélienne et points de torsion." Inventiones Mathematicae 71, no. 1 (1983): 207-33.

[16] Raynaud, M. "Sous-variétés d'une variété abélienne et points de torsion." Arithmetic and Geometry, vol. I, 327-52. Progress in Mathematics 35. Boston, MA: Birkhäuser, 1983.

[17] Scanlon, T. "Automatic uniformity." International Mathematics Research Notices 62 (2004): 3317-26.

[18] Silverman, J. H. "Advanced topics in the arithmetic of elliptic curves." Graduate Texts in Mathematics 151. New York: Springer, 1994.

[19] Szpiro, L., E. Ullmo, and S. Zhang. "Équirépartition des petits points (French) [Uniform distribution of small points]." Inventiones Mathematicae 127, no. 2 (1997): 337-47. 
14 D. Ghioca et al.

[20] Ullmo, E. "Positivité et discrétion des points algébriques des courbes." Annals of Mathematics (2) 147, no. 1 (1998): 167-79.

[21] Yuan, X. "Big line bundles over arithmetic varieties." Inventiones Mathematicae 173 (2008): 603-49.

[22] Yuan, X. and S. Zhang. "Calabi theorem and algebraic dynamics." (2010): preprint http://www.math.columbia.edu/ szhang/papers/cyds.pdf.

[23] Yuan, X. and S. Zhang. "Small points and Berkovich metrics." (2010): preprint http://www. math.columbia.edu/ szhang/papers/spbm.pdf.

[24] Zhang, S. "Small points and adelic metrics." Journal of Algebraic Geometry 4 (1995): 281-300.

[25] Zhang, S. "Equidistribution of small points on Abelian varieties." Annals of Mathematics (2) 147 (1998): 159-65.

[26] Zhang, S. "Distributions in algebraic dynamics, 381-430." Survey in Differential Geometry 10. Somerville, MA: International Press, 2006. 